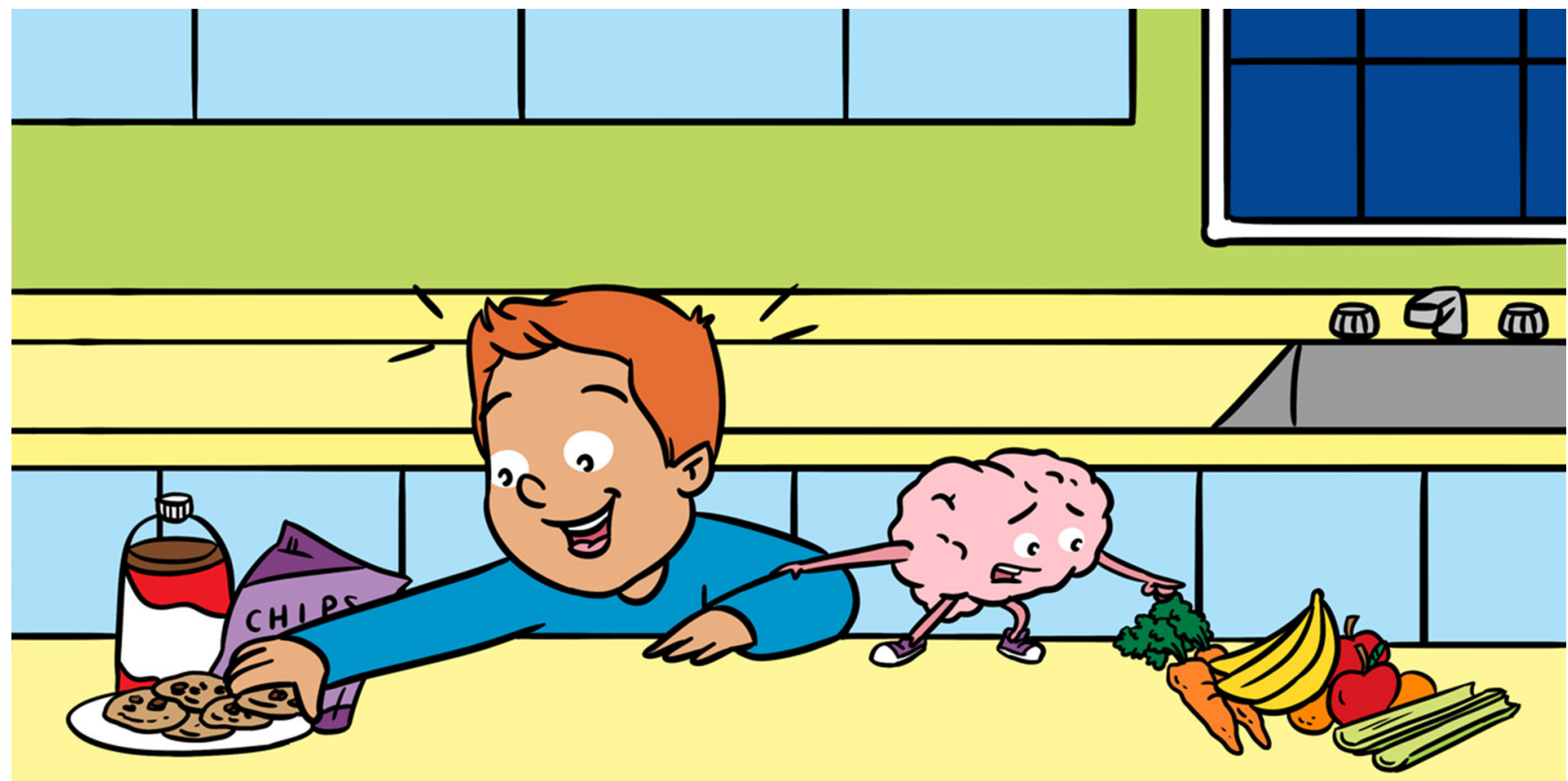

\title{
FOOD FOR THOUGHT: WHAT HAPPENS TO THE BRAIN WHEN WE EAT FOODS HIGH IN FAT AND SUGAR?
}

\section{Erika Calvo-Ochoa ${ }^{1 *}$ and Clorinda Arias ${ }^{2}$}

${ }^{1}$ Department of Biological Sciences, Western Michigan University, Kalamazoo, MI, United States

¿ Departamento de Medicina Genómica y Toxicología Ambiental, Instituto de Investigaciones Biomédicas, Universidad Nacional Autónoma de México, Mexico City, Mexico

\section{YOUNG REVIEWERS:}

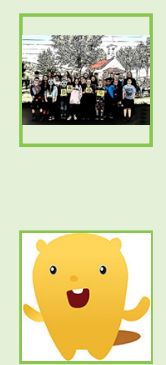

HUFFAKER ELEMENTARY SCHOOL

AGES: $10-11$

MAXWELL

AGE: 12
Have you ever wondered how different foods in your diet affect your brain? The brain is an extraordinary organ in charge of an astounding number of functions in your body. This is why the brain requires highquality nutrients found in the food you eat. But what happens to your brain when you eat processed foods high in fat and sugar? To answer this question, we fed rats a diet high in fat and sugar and then studied its effects in a part of the brain important for memory and learning, known as the hippocampus. Surprisingly, we found out that eating foods high in fat and sugar, even for short periods of time, produces several harmful changes in the hippocampus. We think that these changes could have a negative impact on memory and learning. 


\section{OBESITY}

The condition of being excessively overweight In most cases, obesity is due to eating an unhealthy diet and lack of exercise.

\section{DIABETES}

A disease with elevated levels of glucose in the blood, due to the body's inability to produce the hormone insulin (type 1), or to respond to insulin (type 2).

\section{ALZHEIMER'S}

\section{DISEASE}

A disease characterized by mental deterioration and loss of memory. It is also known as dementia or senility and it is present in old age.

\section{HIPPOCAMPUS}

The brain region in charge of storing memories and learning It is made up of neurons and glial cells.

\section{NEURON}

A nerve cell found in the brain and the spinal cord that receives, processes, and transmits information through electrical and chemical signals.

\section{GLIAL CELLS}

Support cells in the brain. There are two types of glial cells, called astrocytes and microglia. Glial cells nourish, protect and give stability to neurons and become activated to protect neurons from inflammation.

\section{DIETS HIGH IN FAT AND SUGAR AFFECT THE BODY AND THE BRAIN}

You may know that a nutritious diet full of whole grains, fruits, vegetables, dairy, nuts, seeds, and protein is good for your health. This type of diet is not only delicious, but it also makes people grow strong and healthy. In contrast, eating processed foods and beverages high in saturated animal fat and sugar, like processed meats, cookies, candy, sugary drinks, and potato chips, can be very harmful to your health. Did you know that eating these types of foods regularly can cause diseases, such as obesity and type 2 diabetes? [1]. Because processed foods, sugary drinks, and candy are very easy to find and can be tasty (who does not like a candy bar, or a slice of pepperoni pizza?) it is no surprise that obesity and diabetes are two of the most common diseases in the world. Today, around 650 million people are obese, and 422 million people are diabetic-are not these numbers astonishing? Something that is also surprising is that foods high in fat and sugar can do more than make us obese or diabetic-they can also affect our brains! In fact, scientists around the world have noticed that people who are obese or diabetic are more likely to develop memory loss and even dementia or Alzheimer's disease when they get older [2]. Can you believe this?

Fun fact: Did you know that there are two types of diabetes? The most common, type 2 diabetes, occurs when the body gradually stops responding to the hormone insulin. Scientists and physicians believe that this happens because of eating diets high in fats and sugar, and lack of exercise. Type 1 diabetes occurs when someone's pancreas stops producing insulin. Type 1 diabetes is not caused by diet.

\section{THE HIPPOCAMPUS IS THE REGION WHERE MEMORIES ARE STORED IN THE BRAIN}

Did you know that our brains contain highly specialized regions for performing different tasks? For example, there are different areas of the brain for talking, walking, hearing, etc. One of these very specialized regions, known as the hippocampus, is in charge of storing memories and helping us learn [3]. The hippocampus, like all other areas of the brain, is made up of two main types of cells: neurons and glial cells. Neurons in the hippocampus make memories by communicating with one another through very small structures called synapses; these tiny structures are located on long, branch-like structures called dendrites. Did you know that neurons can transmit electrical signals, like the electrical wires you see along the street? These signals are transmitted through the dendrites, and this allows the messages in the brain to travel very quickly. The other types of cells in the brain, glial cells, have 
Figure 1

The hippocampus is the brain region where memories are made and stored. The location of the hippocampus in the rat brain is shown in purple. If we look at the hippocampus under a microscope, we can see that it is made up of neurons and glial cells. There are two types of glial cells: astrocytes and microglia. Neurons have many specialized regions that allow them to receive and send messages and thus communicate with other neurons. Dendrites are long, branch-like structures that transmit electrical signals in the brain. Synapses are tiny structures at the end of dendrites that help neurons communicate.

\section{SYNAPSES}

Small structures used for neuronal communication, located on dendrites.

\section{DENDRITES}

Long, branch-like structures of neurons that transmit signals at a very fast speed.

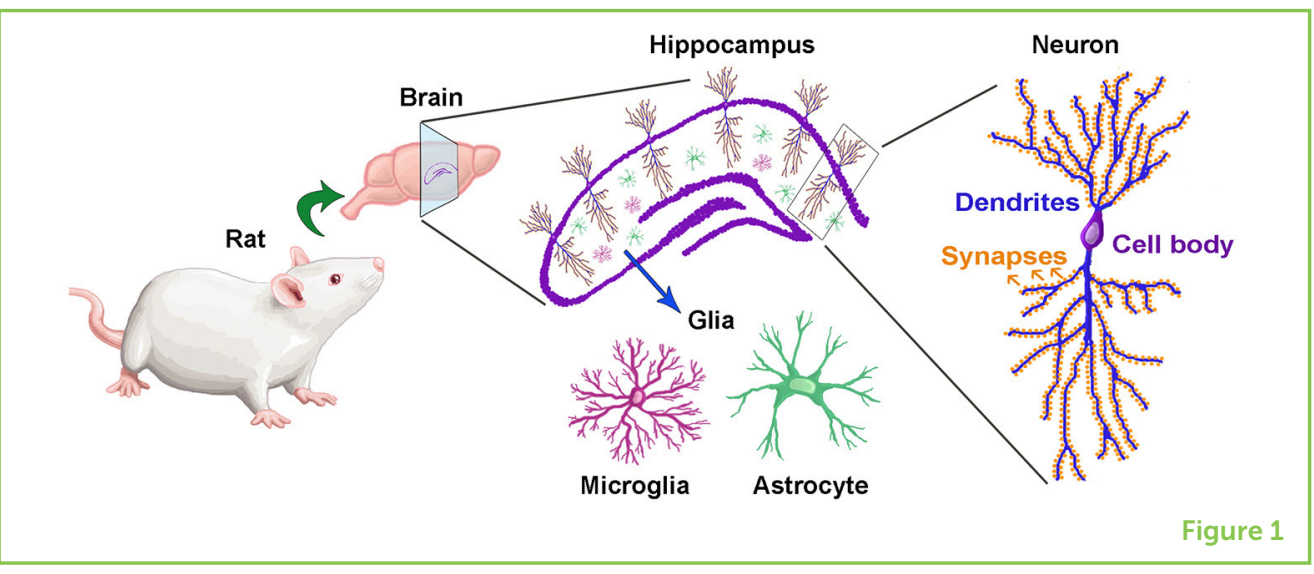

multiple functions. Glial cells nourish, protect, and give stability to neurons. In the hippocampus, both neurons and glial cells are critical for storing memories and helping us learn (Figure 1).

Fun fact: Did you know that, when neurons in the hippocampus get damaged or die, memory loss and learning difficulties arise? Neuron damage can happen after a severe blow to the head, like a concussion, and in some neurodegenerative diseases, such as dementia and Alzheimer's.

\section{OUR EXPERIMENT: STUDYING THE EFFECTS OF FOODS HIGH IN FAT AND SUGAR IN THE BRAINS OF RATS}

We know that eating diets high in fat and sugar can cause obesity and diabetes, and that millions of people worldwide have these diseases. We also know that obesity and diabetes increase the risk of memory loss or dementia as we age. So, we asked the following question: does consuming diets high in fat and sugar affect the brain region that stores memories, the hippocampus? To answer this question, we fed rats a diet high in fat and sugar and did various experiments to look what happened to their hippocampi after they ate this diet. We divided the rats into two groups and gave one group a normal diet (the control group) and the other group a high-fat-and-sugar diet. Having a control group was important, because we needed to compare the hippocampi between rats eating the two diets, in order to see changes. It was also important to have several rats in each group, because the hippocampus may look slightly different in each individual rat. We used 20 rats for our control group and 22 rats for our high-fat/high-sugar group. The control group ate diet consisting of a balanced and nutritious rat food used in laboratories; as well as plain water to drink. The second group ate diet with lard (pork fat used for cooking) added to their normal food, and fructose (the main sugar present in sugary drinks) added to their drinking water. Both groups ate these diets for 7 days, and had as much food and water as they wanted. Rats that ate the high-fat-and-sugar 
diet became obese, ate more food, and drank more water than rats that ate a normal diet. These results show that rats like and consume lots of fatty and sugary foods, and become obese after eating those foods, similar to humans.

\section{EATING A DIET HIGH IN SUGAR AND FAT HAS A NEGATIVE EFFECT ON NEURONS IN THE HIPPOCAMPUS}

For our first experiment, we stained the neurons of the hippocampus with different types of dyes, in order to study their size, shape, and structures under a microscope. We compared rats that ate the highfat-and-sugar diet with rats that ate the normal diet and looked for changes in the neurons (Figure 2 upper half). Astoundingly, we found that neurons from rats that ate the high-fat-and-sugar diet had shorter and thinner dendrites (Figure 2 bottom half, left panel). Amazingly, we also found that the neurons of these rats had fewer synapses at the ends of their dendrites! (Figure 2 bottom half, middle panel). Remember that dendrites and synapses are necessary for neuronal communication and memory formation, because dendrites transmit signals and synapses are the actual sites of neuronal communication. These results showed us that eating high-fat-and-sugar diet had a negative effect on neurons in the hippocampus. What were our conclusions from this experiment? Think about this: if neurons in the hippocampus of rats that ate the high-

Figure 2

We studied what happens to the hippocampus after rats ate a high-fat-and-sugar diet for 7 days (bottom half) and compared it to rats eating a normal diet (upper half):

(1) Neurons have fewer, shorter, and thinner dendrites; (2) Neurons had fewer synapses; and (3) Glial cells became activated by inflammation in the brain. We concluded that eating a diet high in fat and sugar for seven days caused obesity and produced negative effects on the neurons and glial cells of the hippocampus. We believe that these neuronal and glial changes could have a negative effect on memory and learning. fat-and-sugar diet have fewer and thinner dendrites, it is possible that those neurons are not very efficient at transmitting information. Also, because these neurons have fewer synapses, they will probably be unable to communicate effectively with other neurons. We believe that these negative effects of a high-fat-and-sugar diet could result in memory loss and learning problems. How can this happen? The brain is an organ that changes rapidly, constantly forming new synapses as we learn and form memories, and diets high in saturated fats and sugar interfere with the brain's ability to change and adapt quickly. Thus, it is necessary for neurons to receive quality nutrients from a healthy, balanced diet.

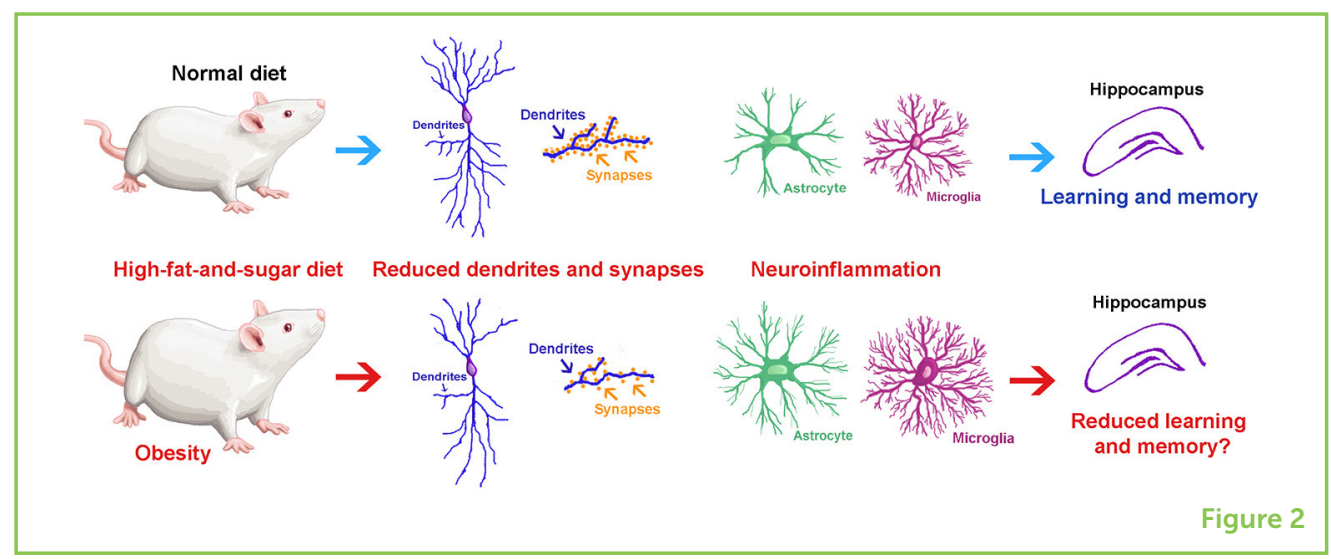




\section{EATING A DIET HIGH IN FAT AND SUGAR CAUSES BRAIN INFLAMMATION}

Do you remember that the hippocampus is made out of two types of cells-neurons and glial cells? Because glial cells are very important to support the function of neurons, we wanted to know if glial cells were also affected by eating a high-fat-and-sugar diet. To do this, we stained two types of glial cells, called astrocytes and microglia, and studied their size and shape. Amazingly, we found that rats that ate the highfat-and-sugar diet had astrocytes and microglia that were larger and had changed to a state that is called "activated." Astrocytes and microglia normally become activated by inflammation, in order to protect neurons from harm. You may be familiar with inflammation and how it looks in your body. Inflammation is a local response to injury or infection. Sometimes the inflamed body part becomes swollen, red, and painful. In the brain, inflammation may look and feel a little different than in other parts of the body, and glial cells are responsible for responding to inflammation in the brain and helping the neurons recover. When astrocytes become activated by inflammation, they multiply and become larger and darker (Figure 2 bottom half, right panel). When microglia gets activated, they also become larger and more branched. What was our conclusion? Our results showed us that eating a high-fat-and-sugar diet produced inflammation in the hippocampus, because astrocytes and microglia became activated in response to the harmful environment.

\section{OUR CONCLUSIONS: A NUTRITIOUS, BALANCED DIET IS FUNDAMENTAL FOR OUR BRAIN HEALTH AND FOR AVOIDING MEMORY LOSS IN THE FUTURE}

In our study, we observed that eating a diet high in fat and sugar, even for very short periods of time, causes obesity and can have negative effects on the hippocampus [4]. Because the hippocampus is an important brain region for memory formation, we believe that these neuronal and glial cell changes could have a negative effect on memory and learning. Other scientists have studied the effects of eating high-fat diets for longer periods of time, and have observed memory loss and learning difficulties in rats and mice. Why are these results important to us? Although we are not rats, we have similarities in the way we process nutrients from food and how these nutrients affect our health. Do you remember that scientists also found that humans who ate highfat diets regularly and became obese or diabetic had a higher chance of experiencing memory loss and dementia? These results could help explain why that happens! Other studies done by us and by other scientists have found that a type of fat called saturated fats, which is very abundant in processed foods, can directly damage neurons 
by changing the way they use energy and by increasing molecules that cause stress and inflammation $[5,6]$. All these amazing scientific studies back up what your parents and doctors always tell you: eating a nutritious, balanced diet low in processed foods and sugar is very important for staying healthy, strong, and smart!

\section{ORIGINAL SOURCE ARTICLE}

Calvo-Ochoa, E., Hernández-Ortega, K., Ferrera, P., Morimoto, S., and Arias, C. 2014. Short-term high-fat-and-fructose feeding produces insulin signaling alterations, dendritic and synaptic loss and astroglial response in the rat hippocampus. J. Cereb. Blood Flow Metab. 34:1001-8. doi: 10.1038/jcbfm.2014.48

\section{REFERENCES}

1. Fung, T. T., Rimm, E. B., Spiegelman, D., Rifai, N., Tofler, G. H., Willett, W. C., et al. 2001. Association between dietary patterns and plasma biomarkers of obesity and cardiovascular disease risk. Am. J. Clin. Nutr. 73:61-7. doi: 10.1093/ajcn/73.1.61

2. Parrott, M. D., and Greenwood, C. E. 2007. Dietary influences on cognitive function with aging: from high-fat diets to healthful eating. Ann. N. Y. Acad. Sci. 1114:389-97. doi: 10.1196/annals.1396.028

3. Neves, G., Cooke, S. F., and Bliss, T. V. 2008. Synaptic plasticity, memory and the hippocampus: a neural network approach to causality. Nat. Rev. Neurosci. 9:65-75. doi: 10.1038/nrn2303

4. Calvo-Ochoa, E. and Arias, C. 2015. Cellular and metabolic alterations in the hippocampus caused by insulin signaling dysfunction and its association with cognitive impairment during aging and Alzheimer's disease. Animal models of study. Diabetes Metab. Res. Rev. 31:1-13. doi: 10.1002/dmrr.2531

5. Kwon, B., Lee, H. K., and Querfurth, H. W. 2014. Oleate prevents palmitateinduced mitochondrial dysfunction, insulin resistance and inflammatory signaling in neuronal cells. Biochim. Biophys. Acta 1843:1402-13. doi: 10.1016/j.bbamcr.2014.04.004

6. Calvo-Ochoa, E., Sánchez-Alegría, K., Gómez-Inclán, C., Ferrera, P., and Arias, C. 2017. Palmitic acid stimulates energy metabolism and inhibits insulin/PI3K/AKT signaling in differentiated human neuroblastoma cells: the role of mTOR activation and mitochondrial ROS production. Neurochem. Int. 110:75-83. doi: 10.1016/j.neuint.2017.09.008

SUBMITTED: 26 July 2018; ACCEPTED: 12 February 2019; PUBLISHED ONLINE: 01 March 2019.

EDITED BY: Vaughan G. Macefield, Baker Heart and Diabetes Institute, Australia 

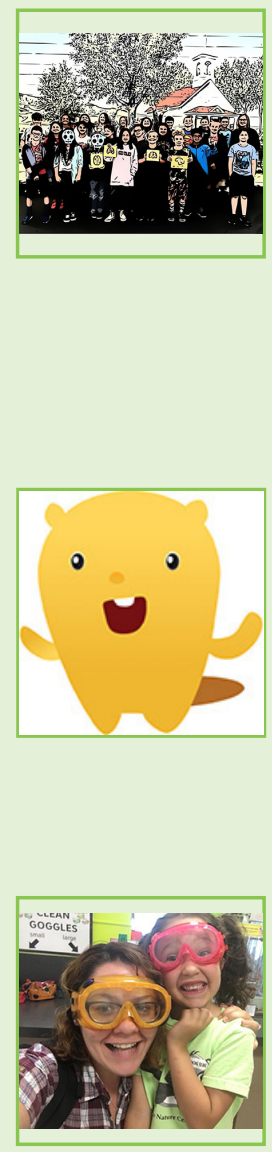

\section{HUFFAKER ELEMENTARY SCHOOL, AGES: 10-11}

This article was reviewed by Ashley Herron M. S. and her sixth grade class at Huffaker Elementary in Reno, Nevada with the help of UNR graduate students and neuroscience mentors Taissa Lytchenko and Grant Fairchild. The class loved learning about the effects of food high in fat and sugar on memory, especially since the mentors brought brain slices with them and showed those areas in the brain during the review. The students were inspired to learn more about the brain and become scientist themselves in the future. They also had a great time reviewing the paper and cannot wait to see it published!

\section{MAXWELL, AGE: 12}

I am living in Beijing and I am interested in science. I like to play video games. I play piano, too. I have no brother or sister. I enjoy reading, about almost everything, not just science. I would like to know something about everything, and everything about something, which I am still looking for.

\section{AUTHORS}

\section{ERIKA CALVO-OCHOA}

I am a Mexican Neuroscientist currently as working as an NSF postdoctoral fellow in Michigan, USA. I am interested in studying how the brain changes after different types of harmful stimuli and how newly born neurons help to repair this damage to the brain. Out of the lab, I love to spend time with my family; we love to hike, grill, watch movies, play board games, go to the lake, and enjoy all seasonal activities in Michigan. *erika.calvoochoa@wmich.edu

\section{CLORINDA ARIAS}

I am a Senior Neuroscientist at the National University of Mexico. I have been working for more than 30 years on brain changes associated with aging and particularly the risk factors for Alzheimer's disease. I love to research the mystery of the incredible human brain. In my free time, I like to read and cook healthy foods using international recipes. 